\title{
A mutant mouse with a highly specific contextual fear-conditioning deficit found in an $\mathrm{N}$-ethyl-N-nitrosourea (ENU) mutagenesis screen
}

\author{
Leon G. Reijmers, ${ }^{1}$ Jennifer K. Coats, ${ }^{1}$ Mathew T. Pletcher, ${ }^{2}$ Tim Wiltshire, ${ }^{2}$ \\ Lisa M. Tarantino, ${ }^{2}$ and Mark Mayford ${ }^{1,3,4}$ \\ ${ }^{1}$ Institute for Childhood and Neglected Diseases, Department of Cell Biology, The Scripps Research Institute, La Jolla, \\ California 92037, USA; ${ }^{2}$ Genomics Institute of the Novartis Research Foundation, San Diego, California 92121, USA; \\ ${ }^{3}$ Department of Neurosciences, University of California at San Diego, La Jolla, California 92093, USA
}

\begin{abstract}
Targeted mutagenesis in mice has shown that genes from a wide variety of gene families are involved in memory formation. The efficient identification of genes involved in learning and memory could be achieved by random mutagenesis combined with high-throughput phenotyping. Here, we provide the first report of a mutagenesis screen that has generated memory mutants in the mouse. We tested a group of N-ethyl-N-nitrosourea (ENU) mutagenized mice in the conditioned fear paradigm. We screened for both dominant and recessive mutations that caused impairments in contextual or tone fear conditioning. Heritability testing confirmed three fear conditioning mutants, i.e., Forgetful, Slowlearner, and Scatterbrain. All three have a learning or short-term memory deficit in contextual fear conditioning. Forgetful was further characterized and showed a highly specific phenotype. The contextual fear-conditioning deficit was apparent when Forgetful was trained with tone-shock pairings, but not when trained with shock alone. The deficit was not due to changes in shock sensitivity or anxiety. Forgetful was not impaired in two other memory tests (hidden platform version of Morris water maze and object recognition). Our data show that a mutagenesis screen can generate mutant mice with highly specific memory phenotypes that can supplement existing mice with targeted mutations. Mapping of Slowlearner found linkage to a region of chromosome 12 (LOD score of 6.5 close to D12Mit171), which suggests that ENU mutants should enable the positional cloning of genes involved in memory formation.
\end{abstract}

Though memory is expressed at the behavioral level, it is dependent on molecular events. Knockout and transgenic mice have shown that a large number of genes are involved in memory formation (Mayford and Kandel 1999; Silva 2003; Tonegawa et al. 2003). Quantitative trait locus analysis in mice has identified many genomic regions that contribute to learning and memory (Caldarone et al. 1997; Wehner et al. 1997; Valentinuzzi et al. 1998; Steinberger et al. 2003). It has become apparent from these studies that memory formation requires the coordinated activity of a large number of genes encoding proteins involved in neurotransmission, signal transduction, regulation of gene expression, and the growth and differentiation of neuronal processes.

Mutagenesis screens that combine random mutagenesis with high-throughput phenotyping provide an efficient method for genetically dissecting complex phenotypes. They can identify critical genetic components, and because of their unbiased character, can lead to the discovery of new mechanisms. Mutagenesis screens using Drosophila have revealed key insights into the genetics of learning and memory (Dubnau and Tully 1998). Complex behavioral phenotypes can also be dissected with mutagenesis screens that use model organisms more closely related to humans, as was demonstrated by the Clock mutant mouse (Vitaterna et al. 1994; King et al. 1997).

Here, we provide the first report of a mutagenesis screen that has generated memory mutants in the mouse. We screened a large number of mice with N-ethyl-N-nitrosourea (ENU)-induced

${ }^{4}$ Corresponding author.

E-mail mmayford@scripps.edu; fax (858) 784-9860.

Article and publication are at http://www.learnmem.org/cgi/doi/10.1101/ Im.98606. point mutations in a test battery that allowed us to recover mutants with highly specific memory phenotypes. Fear conditioning was used as the primary screen for detecting memory phenotypes because it enables high-throughput phenotyping and incorporates different consolidation phases of both hippocampus-independent (tone) and hippocampus-dependent (context) memories (Kim and Fanselow 1992; Phillips and LeDoux 1992). We recovered three mutants with a specific impairment in contextual fear conditioning and analyzed one mutant called Forgetful in more detail. Our data show that a mutagenesis screen can generate mutant mice with highly specific memory impairments that can differ behaviorally from previously reported mutants. The unbiased way in which ENU mutants are generated allows for potential new insights into the mechanisms of memory formation.

\section{Results}

\section{Selection of candidate mutants}

with specific memory phenotypes

We tested 192 wild-type C57BL/6J (B6) males in the fearconditioning protocol that we used as a primary screen (Fig. 1). We obtained the following freezing scores (average percent freezing $\pm \mathrm{SD})$ : baseline $(4 \% \pm 4)$, end of training $(70 \% \pm 20)$, 1 -d context $(74 \% \pm 16)$, 1 -d tone $(69 \% \pm 22)$, 2 -wk context $(63 \% \pm 20), 2$-wk tone $(63 \% \pm 23)$. Based on those wild-type freezing scores, we decided to treat a $G_{3}$ mouse as a candidate mutant when it had a freezing score lower than $20 \%$ for any of those measures except baseline freezing. This resulted in a manageable false positive rate, since only 1\%-3\% of 192 wild-type B6 


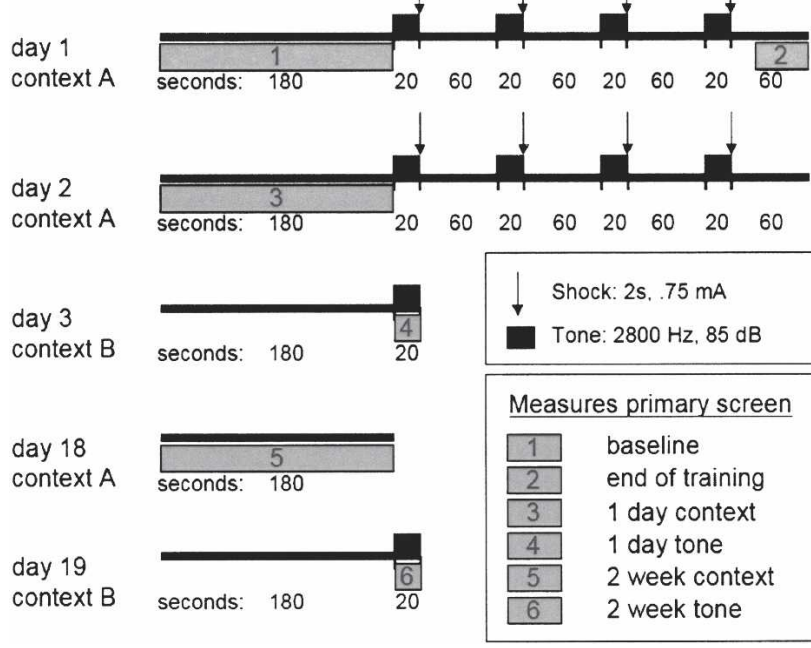

Figure 1. The diagram describes the fear-conditioning protocol that was used as the primary screen. Freezing was measured during the intervals indicated by the gray boxes. This resulted in six measures that were used to screen for candidate mutants among the $\mathrm{G}_{3}$ s.

mice had a freezing score lower than $20 \%$ for any of those measures (except 2-wk tone: $6 \%$ of wild-type $\mathrm{B} 6<20 \%$ freezing). Since $91 \%$ of the wild-type B6 mice had a baseline freezing score lower than $10 \%$, a low occurrence of false negatives was expected.

We screened a total of $6400 \mathrm{G}_{3}$ s derived from $260 \mathrm{G}_{1}$ founders (average of $24 \mathrm{G}_{3}$ mice/family). Since recessive mutations should affect $1 / 8$ of the $\mathrm{G}_{3} \mathrm{~s}$ and dominant mutations should affect $5 / 8$ of the $G_{3} s$, we screened for almost all of the mutations that were present within the 260 families (false negative chance: recessive mutations $P<0.04$; dominant mutations $P<0.0001)$. We found a large number of candidate mutants with impaired fear conditioning $\left(182 \mathrm{G}_{3} \mathrm{~s}\right)$. We reduced false positives by retesting mice in fear conditioning and looking for multiple hits within $\mathrm{G}_{3} \mathrm{~S}$ derived from the same $G_{1}$. Some candidate mutants had phenotypes that were detected in other tests that were part of the primary screen. For example, we found mutants with impaired tone fear conditioning and impaired startle or prepulse inhibition ( 14 of $182 \mathrm{G}_{3} \mathrm{~s}$ ), suggesting a hearing deficit. We also found a number of mutants with gross morphological deficits (35 of $182 \mathrm{G}_{3} \mathrm{~s}$ ). We pursued only those candidate mutants with no other apparent phenotype than a fear-conditioning impairment.

\section{Confirmation of three}

\section{fear-conditioning mutants}

We performed heritability testing on a total of 30 candidate mutants. Candidates could be categorized by the earliest phase when the impairment was detected, i.e., end of training: $n=7 ; 1-\mathrm{d}$ context: $n=19 ; 2$-wk context: $n=4$. All candidate mutants that had normal contextual fear conditioning, but lower 1-d tone or 2-wk tone freezing also had a startle or prepulse inhibition phenotype. Since this suggested a hearing deficit as the primary cause, no heritability testing was done on those candidate mutants.

We tested a minimum of $20 \mathrm{~F}_{2}$ mice for each candidate memory mutant. We confirmed three mutants by recovering the impaired fear conditioning phenotype in the $\mathrm{F}_{2}$ generation. The three mutants were called Slowlearner, Forgetful, and Scatterbrain. Figure 2A,B,C shows the 1-d context freezing for the $\mathrm{F}_{2} \mathrm{~S}$ of the three confirmed mutants. We established homozygous lines by intercrossing affected $\mathrm{F}_{2} \mathrm{~S}$ (freezing $<20 \%$ ). We could not produce Slowlearner $\mathrm{F}_{3} \mathrm{~s}$ because of breeding problems with Slowlearner females. The Forgetful and Scatterbrain $\mathrm{F}_{3} \mathrm{~s}$ showed a high percentage of affected mice (Fig. 2B,C). Homozygosity of the Forgetful $\mathrm{F}_{3} \mathrm{~S}$ was tested by intercrossing $\mathrm{F}_{3} \mathrm{~S}$ with either high or low 1-d context freezing. The offspring of both intercrosses had an identical distribution of 1-d context freezing, suggesting that both low- and high-freezing $\mathrm{F}_{3} \mathrm{~S}$ were homozygous for the Forgetful mutation (Fig. 2D; avg. \pm SEM for offspring of low parents: $26 \% \pm 2.9$; offspring of high-freezing parents: $28 \% \pm 2.8$; $t_{(42)}=-0.39$, n.s.).

\section{The three fear conditioning mutants have a deficit in learning or short-term memory of contextual fear} We maintained homozygous lines for Forgetful and Scatterbrain. After maintaining Slowlearner as a heterozygous line for several generations, we succeeded in obtaining homozygous Slowlearner mice by using BALB/cByJ foster mothers. However, after initial success with foster mothers, the Slowlearner line was discontinued because of persistent breeding problems. We trained homozygous groups of the three mutants and one group of B6. Both Forgetful and Scatterbrain had a significant impairment in learning of fear conditioning (Fig. 3A; two-way ANOVA with repeated measures; main effect genotype: $F_{(3,31)}=5.1, P<0.01$; main effect training: $F_{(8,248)}=82, P<0.001$; interaction $F_{(24,248)}=2.0$, $P<0.01$; post hoc Tukey HSD: $P<0.05$ for Forgetful vs. B6 and Scatterbrain vs. B6). One day after training, all three mutants showed lower 1-d context freezing (Fig. 3B; one-way ANOVA;
A

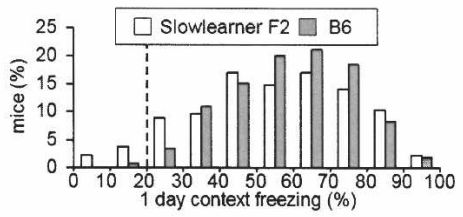

C

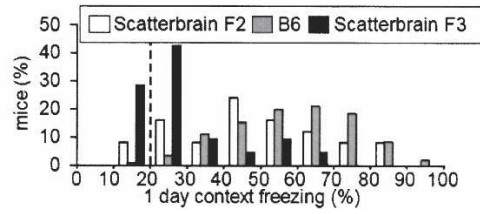

B

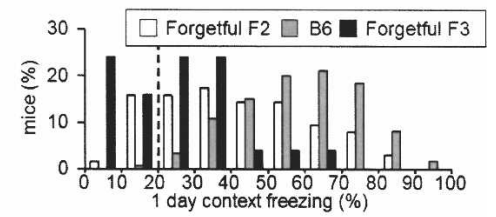

D

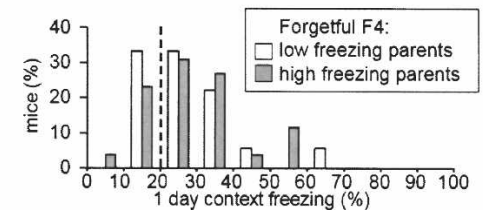

Figure 2. Confirmation of three candidate mutants by heritability testing. The freezing distribution for the 1-d context measure is shown for $\mathrm{F}_{2} \mathrm{~s}, \mathrm{~F}_{3} \mathrm{~s}$, and $\mathrm{F}_{4} \mathrm{~s}$ of the three confirmed mutants. All mice were trained in context $\mathrm{A}$, similar to day 1 of the primary screen, and tested $1 \mathrm{~d}$ later in context $\mathrm{A}$ for $3 \mathrm{~min}$. $(A, B, C)$ Compared with wild-type $B 6$, the $F_{2}$ s of the three mutants had more mice with freezing scores lower than 20\%. $(B, C)$ Putative homozygous $F_{3} s$ of Forgetful and Scatterbrain were produced by intercrossing $F_{2} s$ with freezing scores lower than $20 \%$. The $F_{3}$ mice had a high percentage of mice with freezing scores lower than $20 \%$. (D) To test whether all Forgetful $\mathrm{F}_{3} \mathrm{~s}$ were homozygous, two groups of Forgetful $\mathrm{F}_{4} \mathrm{~s}$ were generated. One group had $\mathrm{F}_{3}$ parents with high 1 -d context freezing scores (38\%-51\%; high-freezing parents), while the other group had $F_{3}$ parents with low 1-d context freezing scores (3\%-13\%; low-freezing parents). Both groups of $\mathrm{F}_{4} \mathrm{~s}$ had an identical distribution of 1 - $\mathrm{d}$ context freezing, suggesting that both low-and high-freezing $\mathrm{F}_{3} \mathrm{~s}$ were homozygous for the Forgetful mutation. Numbers of mice: B6: $n=265$; Slowlearner: $\mathrm{F}_{2} n=135$; Forgetful: $\mathrm{F}_{2} n=63, \mathrm{~F}_{3} n=25, \mathrm{~F}_{4}$ low-freezing parents $n=18, \mathrm{~F}_{4}$ high-freezing parents $n=26$; Scatterbrain $\mathrm{F}_{2} n=25, \mathrm{~F}_{3} n=21$.

\section{Learning \& Memory


main effect genotype: $F_{(3,31)}=31, P<0.001$; post hoc Tukey HSD: $P<0.001$ for Slowlearner vs. B6, Forgetful vs. B6, and Scatterbrain vs. B6).

Since freezing during learning is also affected by the exposure to the tones and shocks, we tested the three mutants in three separate experiments at $15 \mathrm{~min}$ after training to measure shortterm context memory. We found a significant short-term memory deficit in both Slowlearner and Forgetful (Fig. 3C ; twoway ANOVA; main effect experiment: $F_{(2,45)}=5.8, P<0.01$; main effect genotype: $F_{(1,45)}=20, P<0.001$; interaction: $F_{(2,45)}=0.61$, n.s.; post hoc Tukey HSD: $P<0.05$ for Slowlearner vs. B6 and Forgetful vs. B6). The data shown in Figure 3 suggest that all three mutants have a deficit in either learning or short-term memory of contextual fear. We tested the mutants for 1-d tone freezing and found no impairment in any of the three mutants (Fig. 3D; two-way ANOVA; main effect experiment: $F_{(2,45)}=1.1$, n.s.; main effect genotype: $F_{(1,45)}=0.003$, n.s.; interaction $F_{(2,45)}=2.8$, n.s.). There was no significant difference in baseline freezing in context B (range avg. baseline: 1\%-4\%). All three mutants had a shock response identical to B6 as measured by counting the number of quadrant crossings while the mouse was given a 2-sec 0.75 mA shock (data not shown).

\section{The Forgetful mutant has a highly specific phenotype}

Since Forgetful had impaired short-term memory for contextual fear, we wanted to test whether this was due to an impairment in learning of contextual fear. For this we had to train Forgetful without tones. Freezing to tones during training could mask a lack of freezing to the context and thus obscure a deficit in learning of contextual fear. When we trained Forgetful mutants in the fear conditioning paradigm without tones, we found, to our surprise, that learning and 1-h and 1-d context freezing were normal (Fig. 4A; two-way ANOVA with repeated measures; main effect genotype: $F_{(1,6)}=0.54$, n.s.; main effect days: $F_{(11,66)}=21$, $P<0.001$; interaction: $F_{(11,66)}=1.4$, n.s. $)$.

We tested whether the contextual fear conditioning impairment was dependent on the training intensity. We trained mice

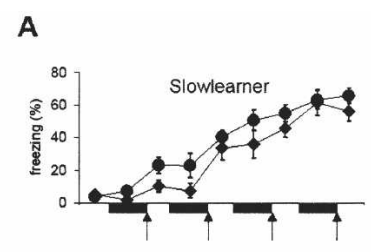

$\mathbf{B}$

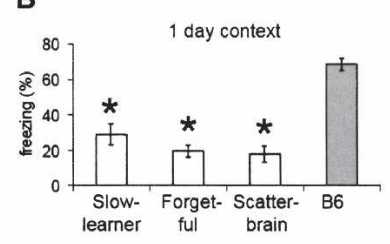

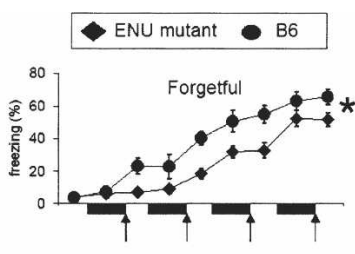

C

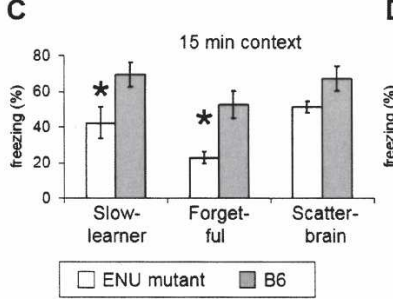

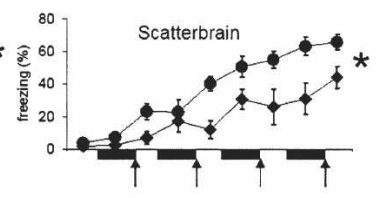

D

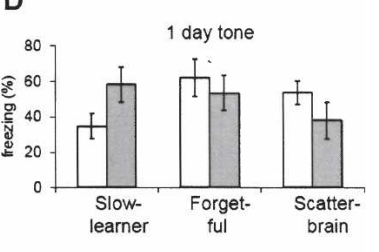

Figure 3. All three mutants have an impairment in learning or short-term memory of contextual fear. $(A)$ Three groups of mutants and one group of B6 were trained in context A, similar to day 1 of the primary screen. Forgetful and Scatterbrain had impaired learning of conditioned fear as compared with B6 $\left({ }^{*} P<0.05 ; \mathrm{B} 6 n=10\right.$, Slowlearner $n=8$, Forgetful $n=14$, Scatterbrain $\left.n=4\right)$. (B) One day after training, all three mutants had lower freezing during a 3-min exposure to context $A\left({ }^{*} P<0.001\right.$; numbers of mice as in $A$ ). (C) Three experiments were done with one mutant and one B6 group per experiment. Mice were trained in context A, similar to day 1 of the primary screen, and returned to the home cage for $15 \mathrm{~min}$, after which they were tested in context A for $3 \mathrm{~min}$. During this test, Slowlearner and Forgetful had lower freezing scores $\left({ }^{*} P<0.05\right.$; Slowlearner experiment: mutant $n=10, \mathrm{~B} 6 n=8$; Forgetful experiment: mutant $n=8, \mathrm{~B} 6 n=7$; Scatterbrain experiment: mutant $n=10, \mathrm{~B} 6 n=8)$. $(D)$ One day after training, the same mice were tested in context B, similar to day 3 of the primary screen. During the presentation of the tone, all three mutants showed normal freezing (numbers of mice as in $C$ ). with only 1-tone shock-pairing per day and found that Forgetful mice were impaired after $4 \mathrm{~d}$ of 1-tone shock training (Fig. 4B; two-way ANOVA with repeated measures; main effect genotype: interaction: $F_{(4,88)}=8.8, P<0.001 ; t$-test between Forgetful and on days $2-4, P<0.05$ on day 5 ). foctive last block (Fig. 4C; two-way ANOVA with repeated measures; main (1,26) $=1.0$, n.s.; main effect blocks: $F_{(3,78)}=25$ getful and B6: $P<0.01$ for block 4). Forgetful and B6 mice showed equal avoidance of the center of the open field (Fig. 4D; two-way ANOVA with repeated measures; main effect genotype: $F_{(1,26)}=0.24$, n.s.; main effect
teraction: $F_{(3,78)}=0.84$, n.s).

\section{The Forgetful mutant is unimpaired in the Morris water} memory, we tested Forgetful in the Morris water maze and in the trials with a cued stationary platform, followed by 10 trials with a hidden platform at the same location as the cued platform. Forgetful was slower than B6 in learning to locate the cued stationary platform (Fig. 5A; two-way ANOVA with repeated meamain effect trials: $F_{(7,161)}=9.2, P<0.001$; main effect genotype: $F_{(1,23)}=10, P<0.01$; interaction: $F_{(7,161)}=1.5$, n.s.; $t$-test the $2 . P<0.01 ; t$-test trial $3: P<0.05)$. Latency times for locating 5A; two-way ANOVA with repeated measures; main effect trials: $F_{(9,207)}=1.1$, n.s.; main effect genotype: $F_{(1,23)}=0.75$, n.s.; interaction: $F_{(9,207)}=0.85$, n.s.). At the beginning of days 3 and 4 , we den platform. The data for those two probe trials were combined, as there were no significant differences between them. Forgetful and B6 mice showed a similar preference for the training quadrant as compared with the opposite quadrant (Fig. 5B; two-way ANOVA with repeated measures; main effect training quadrant vs. opposite quadrant: $F_{(1,23)}=30$, $P<0.001 ;$ main effect genotype: $F_{(1,23)}=3.9$, n.s.; interaction: $F_{(1,23)}=0.55$, n.s.)

In the object-recognition test, Forgetful and B6 mice spent equal amounts of time exploring the objects (data not shown), and showed equal preference for the new object at $1 \mathrm{~h}$ and $1 \mathrm{~d}$ after training (Fig. 5C; two-way ANOVA with repeated measures; main effect interval: $F_{(1,26)}=2.4$, n.s.; main effect genotype $F_{(1,26)}=0.055$, n.s.; interaction: $F_{(1,26)}=1.5$, n.s.).

\section{Slowlearner is linked to chromosome 12}

We mapped the chromosomal location of Slowlearner by linkage analysis using the $F_{2}$ offspring of a (Slowlearner $\times$ BALB/cByJ) F1 intercross (Fig. 6A). We performed a genome-wide search for differential allelic representation by pool- 
A

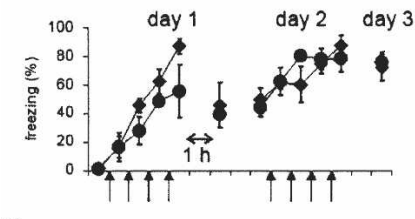

C

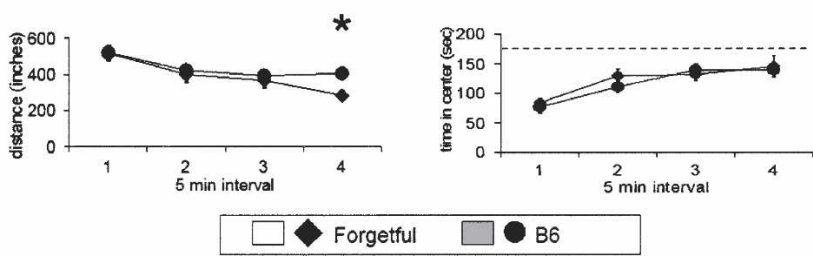

Figure 4. Forgetful has a highly specific phenotype. (A) Forgetful and B6 mice were trained and tested in context A. Training was similar to day 1 of the primary screen, except that no tones were played. One hour after the end of training, mice were tested for $3 \mathrm{~min}$. On day 2, mice were trained again without tones and tested for $3 \mathrm{~min}$ on day 3 . Training of Forgetful in fear conditioning without tone "rescued" the contextual fearconditioning deficit (Forgetful $n=4, \mathrm{~B} 6 n=4$ ). (B) In a separate experiment, Forgetful and B6 mice were placed in context A for 3 min, after which they were given a 1 -tone shock pairing. This was repeated for five consecutive days. Freezing was measured during the first 3 min of each day. Forgetful showed lower freezing on days $2-5\left({ }^{*} P<0.05\right.$; Forgetful $n=12, B 6 n=12)$. $(C, D)$ The open-field behavior of Forgetful was similar to $B 6$ with regard to total distance traveled and time spent in center, with the exception of lower distance traveled during the $4^{\text {th }}$ block of 5 min ( ${ }^{*} P<0.01$; Forgetful $n=12, \mathrm{~B} 6 n=16$ ). The dashed line in $D$ indicates the time spent in center for a mouse that would not avoid the center.

ing DNA from selected individuals at each end of the phenotypic distribution (Lander and Botstein 1989; Darvasi and Soller 1994). We made DNA pools containing DNA from $\mathrm{F}_{2}$ mice with either low or high 1-d context freezing and used single-nucleotide polymorphisms (SNPs) covering the whole genome. We found differential allelic representation on chromosome 12 (Fig. 6B). Chromosome 3 showed a reversed differential allelic representation, with less B6 alleles in the low freezers. This probably reflects a previously reported quantitative trait locus (QTL) that causes higher freezing to context in the B6 strain (Caldarone et al. 1997; Wehner et al. 1997).

Genotyping of the 40 individual $\mathrm{F}_{2}$ mice confirmed linkage to chromosome 12 (LOD = 3.3 for D12Mit285). We tested linkage to parts of other chromosomes that showed a small differential allelic representation (chromosomes 1, 6, 14, and 19), but none reached significance (LOD scores $<1.5$ ). Interval mapping was performed on an extended panel of $\mathrm{F}_{2}$ mice with either low or high 1-d context freezing. We used seven Mit markers spanning the region on chromosome 12 that showed differential allelic representation in the SNP data set. Significant linkage was ob- served with a maximum LOD score of 6.5 close to D12Mit171 (Fig. 6C).

To further test the hypothesized location of the Slowlearner mutation on chromosome 12 , we generated $\mathrm{F}_{2}$ s by intercrossing (Slowlearner $\times 129 \mathrm{~S} 1 / \mathrm{SvImJ}) \mathrm{F}_{1}$ s. This time we genotyped all 204 $\mathrm{F}_{2} \mathrm{~S}$ using the D12Mit171 marker and confirmed significant linkage of Slowlearner to D12Mit171 (LOD score of 6.6). To test the possibility of a B6 QTL on chromosome 12 , we genotyped $66 \mathrm{~F}_{2}$ offspring of a wild-type $(\mathrm{B} 6 \times \mathrm{BALB} / \mathrm{cByJ}) \mathrm{F}_{1}$ intercross (phenotypes shown in Fig. 6A). No linkage was observed between 1-d context freezing and a marker located between D12Mit112 and D12Mit285 (D12Mit64: LOD = 0.3).

\section{Discussion}

Forgetful has a highly specific and unique phenotype We found three ENU mutants with an impairment in learning or short-term memory of contextual fear. The impairment in contextual fear conditioning was not due to lower shock sensitivity, as the response of all three mutants to the shock was similar to B6. Also, all three mutants could be conditioned to tone, indicating normal shock sensitivity and normal ability to express fear by freezing.

We phenotyped the Forgetful mutant in more detail. The phenotype of Forgetful is only exposed if training occurs to context and tone simultaneously. Phillips and LeDoux (1994) have found a similar phenotype after electrolytic lesion of the dorsal hippocampus. The authors suggested that contextual fear conditioning without simultaneous conditioning to the tone might allow elements of the context (odor, grid floor, visual cues) to enter into a direct association with the shock. This association would take place in the amygdala without requiring the hippocampus for processing of the context. It is tempting to speculate that the similarity of the Forgetful phenotype with the hippocampal lesion phenotype is due to impaired hippocampal functioning in Forgetful mice. However, the data from the objectrecognition test and the hidden platform version of the Morris water maze, both found to be hippocampus dependent (Morris et al. 1982; Clark et al. 2000; however, Winters et al. 2004), do not suggest a severe hippocampal impairment in Forgetful mutants. Also, preliminary data from Nissl staining suggest that the general structure of the Forgetful hippocampus is normal (data not shown).

In general, knockout or transgenic mice with a fearconditioning phenotype either display a deficit in both context and tone fear conditioning (for example, Brambilla et al. 1997), or a deficit in contextual fear conditioning that is accompanied by a deficit in the hidden platform version of the Morris water maze (for example, Abel et al. 1997). A specific contextual fear conditioning deficit that is only revealed when training occurs to context and tone simultaneously has, to our knowledge, not
A

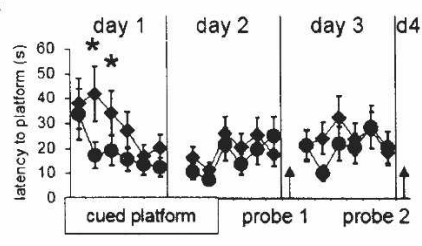

B

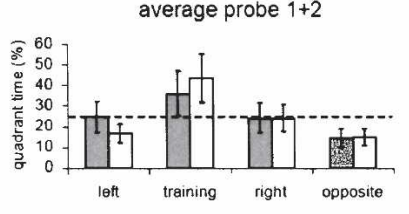

C

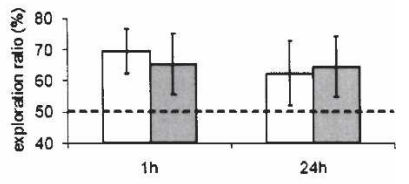

Forgetful

B6

Figure 5. Forgetful is normal in two other memory tests. $(A)$ Forgetful was similar to B6 mice in the Morris water maze with regard to latency to find a cued and subsequently hidden platform, with the exception of trials 2 and $3\left({ }^{\star} P<0.05\right.$; Forgetful $n=11$, B6 $\left.n=14\right)$. (B) During probe trials, both $P<0.05$; Forgetful and B6 mice showed a preference for the quadrant that contained the platform (numbers of mice as in $A$ ). ( $C$ ) Object recognition with 1 and 24-h intervals was similar for Forgetful and B6 mice (Forgetful $n=12$, B6 $n=16$ ). 
A
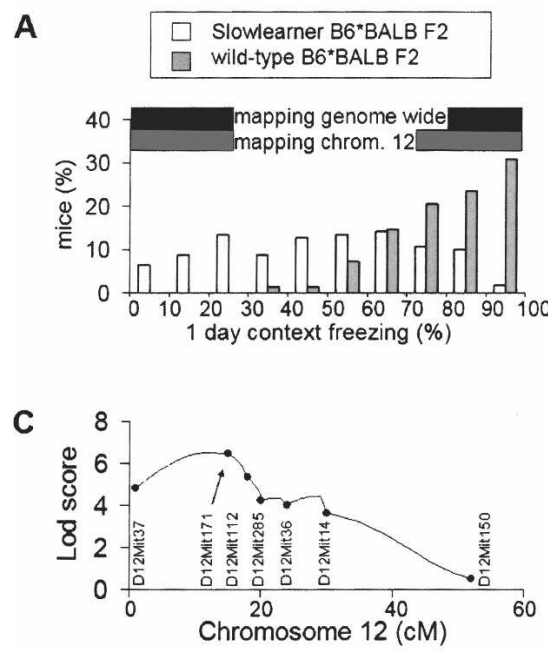

\section{B}

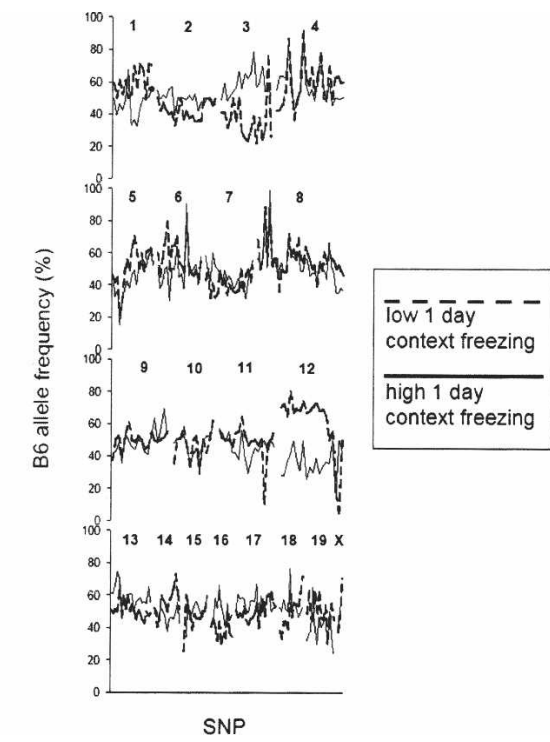

Figure 6. Slowlearner links to chromosome 12. Genome-wide and interval mapping was performed on $\mathrm{F}_{2}$ offspring of a (Slowlearner $\left.\times \mathrm{BALB} / \mathrm{CBy}\right]$ ) $\mathrm{F}_{1}$ intercross. $(A) \mathrm{F}_{2} \mathrm{~s}$ were trained in context $\mathrm{A}$, similar to day 1 of the primary screen, and freezing was measured $1 \mathrm{~d}$ later for $3 \mathrm{~min}$ in context $\mathrm{A}$ (Slowlearner $\mathrm{B} 6 * \mathrm{BALB} \mathrm{F}_{2} n=339$, wild-type $\left.\mathrm{B} 6{ }^{*} \mathrm{BALB} \mathrm{F}_{2} n=66\right)$. (B) For genome-wide mapping we used pooled DNA from $F_{2}$ mice with either low ( $<30 \%$ freezing; $\left.n=20\right)$ or high ( $>80 \%$ freezing; $\left.n=20\right)$ freezing scores for 1-d context freezing. We used SNPs covering the whole genome with an average interval of $7 \mathrm{Mb}$ between consecutive SNPs, and found linkage to chromosome 12 (numbers in graph are chromosome numbers). (C) Interval mapping was performed on an extended panel of $F_{2}$ mice with either low ( $<30 \%$ freezing; $n=35$ ) or high ( $>70 \%$ freezing; $n=29) 1-d$ context freezing using seven MIT markers. The position of the anchor marker D12Mit37 was taken from the NCBI Web site. The other map positions and LOD scores were calculated using Map Manager QTX.

been reported for a mouse with a targeted mutation. Since Forgetful does not resemble any reported mutant, it may lead to new mechanisms for memory formation.

\section{The three mutants have impaired contextual fear conditioning, but normal tone fear conditioning}

All three ENU mutants have a deficit in contextual, but not tone fear conditioning. The higher vulnerability of contextual fear conditioning as opposed to tone fear conditioning might be explained by the different circuits underlying those two forms of memory. Contextual fear conditioning is dependent on the hippocampus and the amygdala, while tone fear conditioning is dependent on the amygdala, but not the hippocampus (Kim and Fanselow 1992; Phillips and LeDoux 1992). A higher vulnerability of the hippocampus, as compared with the amygdala, to the effects of ENU mutations could explain our findings. It is also possible that both hippocampus and amygdala are mildly impaired by an ENU mutation, and that those mild impairments are only revealed when those two brain structures interact with each other, as is the case for contextual fear conditioning. It should be noted that we do not know whether the contextual fear conditioning impairment that we observe in our three mutants is directly related to the hippocampus. Because of the unbiased character of ENU mutagenesis screens, it is possible that ENU mutations tap into new and unanticipated mechanisms.

\section{The number of genes essential for fear conditioning might be limited}

A major benefit of mutagenesis screens is their ability to identify critical components within a complex genetic pathway. The identification of ENU mutants with a complete loss of fear conditioning could lead to the discovery of genes that are essential for fear conditioning. Complete loss of fear conditioning would result in freezing scores equal to baseline freezing (average of $4 \%$ ) during the 1 -d/2-wk context or tone tests. However, our three mutants showed average freezing of at least $18 \%$ during those tests. It is possible that the ENU mutations present within our 260 families were outside the genes that are essential for fear conditioning. We measured the total number of ENU mutations by sequencing $12 \mathrm{Mb}$ of DNA from our $G_{1}$ mice covering different genomic regions. This revealed $\sim 1$ base change per $400 \mathrm{~Kb}$. This indicates a relatively high mutagenic load, as a previous ENU mutagenesis screen reported 1 base change per $1 \mathrm{Mb}$ (Nolan et al. 2000; Quwailid et al. 2004). A previous study found that the ENU doses used in our study result in approximately one functional mutation in every 1000 genes of a single $G_{1}$ (Hitotsumachi et al. 1985). Assuming the presence of 30,000 genes in the mouse genome (Mouse Genome Sequencing Consortium 2002), this would mean that our 260 families contained one functional mutation in $23 \%$ of all genes and two separate functional mutations in about $1 \%-2 \%$ of the genes. Our estimate of the achieved genome coverage is supported by data from an ENU screen, in which B6 males were mutagenized in a similar way as in our study. This screen achieved an estimated $18 \%$ genome coverage in 185 families when T-cell abnormalities were used as a phenotype (Papathanasiou and Goodnow 2005). Despite significant coverage of the genome, we did not find mutants with a complete loss of fear conditioning. This suggests that a relatively limited number of genes are essential for the formation and expression of conditioned fear without being involved in other processes like brain development (see below).

\section{Pleiotropic effects of ENU mutations might prevent detection of memory phenotypes}

As ENU mutations are present throughout development, there is a considerable chance of pleiotropic effects. For example, in our screen we did not find mutants with specific impairments in long-term memory. This might be caused by pleiotropic effects of mutations in genes that are involved in neuronal and synaptic growth. Those mutations could prevent long-term memory consolidation, but they would disrupt the development of the brain before the mice can be tested for fear conditioning. On a similar note, the absence of mutants with a complete loss of fear conditioning might be caused by pleiotropic effects of mutations in genes essential for fear conditioning. Conditional knockouts of genes involved in both brain development and adult memory might be necessary in order to establish their role in fear conditioning.

\section{ENU mutants can be mapped using}

\section{a memory phenotype}

We have linked Slowlearner to chromosome 12 in two separate mapping crosses, using BALB/cByJ and 129S1/SvImJ as mapping strains. It is highly unlikely that the linkage to chromosome 12 was caused by a quantitative trait locus (QTL). We found no 
indication of a QTL within a group of wild-type B6* BALB/cByJ F2 mice, and no contextual fear-conditioning QTL has been reported for chromosome 12 (Caldarone et al. 1997; Wehner et al. 1997; Valentinuzzi et al. 1998). Though no fear-conditioning QTL has been reported on chromosome 12 , there is a report of a spatial memory QTL on chromosome 12 (Steinberger et al. 2003). This suggests the possibility of a gene on chromosome 12 that is involved in both spatial memory and contextual fear conditioning. Our data strongly suggest that Slowlearner is the result of a single mutation that is located on chromosome 12 . While unlikely, it is possible that multiple linked ENU mutations within the mapped region cause the phenotype.

In summary, we have performed an ENU mutagenesis screen covering $\sim 25 \%$ of the genome and have found three mutants with a specific impairment in fear conditioning. The main finding of our study is that ENU mutations can result in highly specific memory phenotypes, as demonstrated by the Forgetful mutant. The Forgetful phenotype is different from the phenotypes of previously reported mutant mice. This suggests that unbiased genetic approaches like ENU mutagenesis screens can supplement model-driven genetic approaches and might result in new insights into the mechanisms of learning and memory. Our mapping data for Slowlearner show that the memory phenotype of an ENU mutant can be mapped to a single chromosome, and suggest that ENU mutants should enable positional cloning of genes involved in memory formation.

\section{Materials and Methods}

\section{ENU mutagenesis and primary screen}

All ENU mutant mice were generated and maintained against a pure $\mathrm{C} 57 \mathrm{BL} / 6 \mathrm{~J}$ background (B6). Male $\mathrm{G}_{0}$ mice were treated four times with $85 \mathrm{mg} / \mathrm{kg}$ ENU as previously described (Hitotsumachi et al. 1985). $G_{0}$ males were bred to wild-type females. The resulting $G_{1}$ males were bred to wild-type females to produce $G_{2}$ females, which were back-crossed to their $G_{1}$ fathers. The resulting $G_{3}$ males were phenotyped in a primary screen that consisted of the following tests (with age at which mice were tested): general developmental deficits ( $4 \mathrm{wk})$, locomotor activity ( $8 \mathrm{wk})$, prepulse inhibition and startle (8 wk), fear conditioning (9 and 11 wk), circadian rhythm (10-11 wk), and pain perception (11 wk). Additional fear-conditioning experiments were done on 7-9-wkold males and females. Open field, water maze, and object recognition tests were done using 8-12-wk-old Forgetful males and females.

\section{Fear conditioning protocol used as primary screen}

Figure 1 summarizes the fear-conditioning protocol used as the primary screen. On day 1 , the $G_{3}$ males were subjected to a training trial by placing them in a lemon-scented square chamber with grid-floor (context A; 30-cm length $\times 24$-cm width; Med Associates). After $3 \mathrm{~min}$ of baseline freezing measurement, the first of four tone-shock pairings was presented. Each tone-shock pairing consisted of a $20-\mathrm{sec} 85-\mathrm{dB} 2800-\mathrm{Hz}$ tone simultaneously ending with a 2 -sec $0.75-\mathrm{mA}$ shock. There was a 1-min interval between each tone-shock pairing. Mice remained in the chamber for $1 \mathrm{~min}$ after the last tone-shock before being returned to the home cage. Freezing was measured during the last $40 \mathrm{sec}$ at the end of the training. On day 2 , the training trial of day 1 was repeated, and the first 3 min were used to measure 1-d context freezing. On day 3 , mice were tested for 1-d tone freezing in the same chamber with all of the context cues being changed (context B; triangular shape, flat floor covered with bedding, wintergreen scent). After a 3-min baseline, the 20 -sec tone was presented and freezing during the tone was measured. On day 18 , the mice were placed in context A for 3 min to measure 2-wk context freezing. On day 19 , mice were placed in context $\mathrm{B}$, and the trial given on day 3 was repeated to measure 2 -wk tone freezing.
Freezing behavior was measured using a digital camera connected to a computer with Actimetrics FreezeFrame software (Actimetrics). The freezing scores attained with the software (minimum bout length $=1 \mathrm{sec}$ ) were validated by comparing them with human-scored freezing, using mice with freezing scores that ranged from $4 \%$ to $90 \%\left(\mathrm{R}^{2}=0.8446 ; t_{(16)}=9.3, P<0.001\right)$.

\section{Open field, water maze, and object recognition}

Mice were placed in an open field $(43-\mathrm{cm}$ length $\times 43-\mathrm{cm}$ width; center defined as area of $33 \mathrm{~cm} \times 33 \mathrm{~cm}$ in middle of open field) for $20 \mathrm{~min}$, and activity was measured using infrared beams and software (Med Associates). Water-maze testing was done in a circular tank (120-cm diam.) filled with opaque water at a temperature of $25^{\circ} \mathrm{C}$. The tank was located in a square room with visible cues attached to the wall at a distance of 1-2 meters from the edge of the tank. The platform was $1 \mathrm{~cm}$ under the water surface and invisible to the mice except during cued training trials, when a $9-\mathrm{cm}^{2}$ flag was placed on the platform using a $10-\mathrm{cm}$ long pole. For each trial, the mouse was gently placed in the water facing the wall of the tank at semirandom starting locations. Maximum trial duration was $60 \mathrm{sec}$, after which the mouse was guided to the platform (61-sec latency was scored), where it stayed for 30 $\mathrm{sec}$ at the end of each trial. The intertrial interval was $1 \mathrm{~h}$. For the probe trials at the beginning of days 3 and 4, the platform was removed and mice were observed for time spent in the four quadrants for a total duration of $60 \mathrm{sec}$. For object recognition, we used objects that varied in color, material, shape, and texture, and that were counterbalanced. An object was placed in the home cage (2-4 mice/cage) and removed after $1 \mathrm{~h}$ for an interval of $1 \mathrm{~h}$ or $1 \mathrm{~d}$. Just before being tested for object recognition, the mice were removed from their home cage and temporarily housed in a clean cage. Each mouse was then individually put in its home cage, which contained the training object and a new object, and exploration of both objects was measured until the mouse had explored for a total of $20 \mathrm{sec}$. Object recognition was calculated as exploration of training object / (exploration of training object + exploration of new object) * $100 \%$.

\section{Linkage analysis}

Slowlearner mutants were bred to either BALB/cByJ or 129S1/ SvImJ, and the resulting $\mathrm{F}_{1} \mathrm{~s}$ were intercrossed. Both male and female $\mathrm{F}_{2} \mathrm{~S}$ were phenotyped in the fear-conditioning test. Genomic DNA was purified from tails and genotyped using SNPs (Wiltshire et al. 2003) or Mit markers. Map Manager QTX (Manly et al. 2001; www.mapmanager.org) was used to calculate LOD scores (likelihood ratio statistic divided by 4.61) and perform interval mapping.

\section{References}

Abel, T., Nguyen, P.V., Barad, M., Deuel, T.A., Kandel, E.R., and Bourtchouladze, R. 1997. Genetic demonstration of a role for PKA in the late phase of LTP and in hippocampus-based long-term memory. Cell 88: 615-626.

Brambilla, R., Gnesutta, N., Minichiello, L., White, G., Roylance, A.J., Herron, C.E., Ramsey, M., Wolfer, D.P., Cestari, V., Rossi-Arnaud, C. et al. 1997. A role for the Ras signalling pathway in synaptic transmission and long-term memory. Nature 390: 281-286.

Caldarone, B., Saavedra, C., Tartaglia, K., Wehner, J.M., Dudek, B.C., and Flaherty, L. 1997. Quantitative trait loci analysis affecting contextual conditioning in mice. Nat. Genet. 17: 335-337.

Clark, R.E., Zola, S.M., and Squire, L.R. 2000. Impaired recognition memory in rats after damage to the hippocampus. J. Neurosci. 20: $8853-8860$

Darvasi, A. and Soller, M. 1994. Selective DNA pooling for determination of linkage between a molecular marker and a quantitative trait locus. Genetics 138: 1365-1373.

Dubnau, J. and Tully, T. 1998. Gene discovery in Drosophila: New insights for learning and memory. Annu. Rev. Neurosci. 21: 407-444.

Hitotsumachi, S., Carpenter, D.A., and Russell, W.L. 1985. Dose-repetition increases the mutagenic effectiveness of N-ethyl-N-nitrosourea in mouse spermatogonia. Proc. Natl. Acad. Sci. 82: 6619-6621.

Kim, J.J. and Fanselow, M.S. 1992. Modality-specific retrograde amnesia of fear. Science 256: 675-677. 
King, D.P., Zhao, Y., Sangoram, A.M., Wilsbacher, L.D., Tanaka, M., Antoch, M.P., Steeves, T.D., Vitaterna, M.H., Kornhauser, J.M., Lowrey, P.L., et al. 1997. Positional cloning of the mouse circadian clock gene. Cell 89: 641-653.

Lander, E.S. and Botstein, D. 1989. Mapping mendelian factors underlying quantitative traits using RFLP linkage maps. Genetics 121: 185-199.

Manly, K.F., Cudmore Jr., R.H., and Meer, J.M. 2001. Map Manager QTX, cross-platform software for genetic mapping. Mamm. Genome 12: 930-932.

Mayford, M. and Kandel, E.R. 1999. Genetic approaches to memory storage. Trends Genet. 15: 463-470.

Morris, R.G., Garrud, P., Rawlins, J.N., and O'Keefe, J. 1982. Place navigation impaired in rats with hippocampal lesions. Nature 297: 681-683.

Mouse Genome Sequencing Consortium. 2002. Initial sequencing and comparative analysis of the mouse genome. Nature 420: 520-562.

Nolan, P.M., Peters, J., Strivens, M., Rogers, D., Hagan, J., Spurr, N., Gray, I.C., Vizor, L., Brooker, D., Whitehill, E., et al. 2000. A systematic, genome-wide, phenotype-driven mutagenesis programme for gene function studies in the mouse. Nat. Genet. 25: 440-443.

Papathanasiou, P. and Goodnow, C.C. 2005. Connecting mammalian genome with phenome by ENU mouse mutagenesis: Gene combinations specifying the immune system. Annu. Rev. Genet. 39: 241-262.

Phillips, R.G. and LeDoux, J.E. 1992. Differential contribution of amygdala and hippocampus to cued and contextual fear conditioning. Behav. Neurosci. 106: 274-285.

- 1994. Lesions of the dorsal hippocampal formation interfere with background but not foreground contextual fear conditioning. Learn. Mem. 1: 34-44.

Quwailid, M.M., Hugill, A., Dear, N., Vizor, L., Wells, S., Horner, E., Fuller, S., Weedon, J., McMath, H., Woodman, P., et al. 2004. A gene-driven ENU-based approach to generating an allelic series in any gene. Mamm. Genome 15: 585-591.

Silva, A.J. 2003. Molecular and cellular cognitive studies of the role of synaptic plasticity in memory. J. Neurobiol. 54: 224-237.

Steinberger, D., Reynolds, D.S., Ferris, P., Lincoln, R., Datta, S., Stanley, J., Paterson, A., Dawson, G.R., and Flint, J. 2003. Genetic mapping of variation in spatial learning in the mouse. J. Neurosci. 23: 2426-2433.

Tonegawa, S., Nakazawa, K., and Wilson, M.A. 2003. Genetic neuroscience of mammalian learning and memory. Philos. Trans. $R$. Soc. Lond. B Biol. Sci. 358: 787-795.

Valentinuzzi, V.S., Kolker, D.E., Vitaterna, M.H., Shimomura, K., Whiteley, A., Low-Zeddies, S., Turek, F.W., Ferrari, E.A., Paylor, R., and Takahashi, J.S. 1998. Automated measurement of mouse freezing behavior and its use for quantitative trait locus analysis of contextual fear conditioning in (BALB/CJ x C57BL/6J)F2 mice. Learn. Mem. 5: 391-403.

Vitaterna, M.H., King, D.P., Chang, A.M., Kornhauser, J.M., Lowrey, P.L., McDonald, J.D., Dove, W.F., Pinto, L.H., Turek, F.W., and Takahashi, J.S. 1994. Mutagenesis and mapping of a mouse gene, Clock, essential for circadian behavior. Science 264: 719-725.

Wehner, J.M., Radcliffe, R.A., Rosmann, S.T., Christensen, S.C., Rasmussen, D.L., Fulker, D.W., and Wiles, M. 1997. Quantitative trait locus analysis of contextual fear conditioning in mice. Nat. Genet. 17: 331-334.

Wiltshire, T., Pletcher, M.T., Batalov, S., Barnes, S.W., Tarantino, L.M., Cooke, M.P., Wu, H., Smylie, K., Santrosyan, A., Copeland, N.G., et al. 2003. Genome-wide single-nucleotide polymorphism analysis defines haplotype patterns in mouse. Proc. Natl. Acad. Sci. 100: 3380-3385.

Winters, B.D., Forwood, S.E., Cowell, R.A., Saksida, L.M., and Bussey, T.J. 2004. Double dissociation between the effects of peri-postrhinal cortex and hippocampal lesions on tests of object recognition and spatial memory: Heterogeneity of function within the temporal lobe. J. Neurosci. 24: 5901-5908.

Received May 27, 2005; accepted in revised form December 7, 2005.
Learning \& Memory www.learnmem.org 


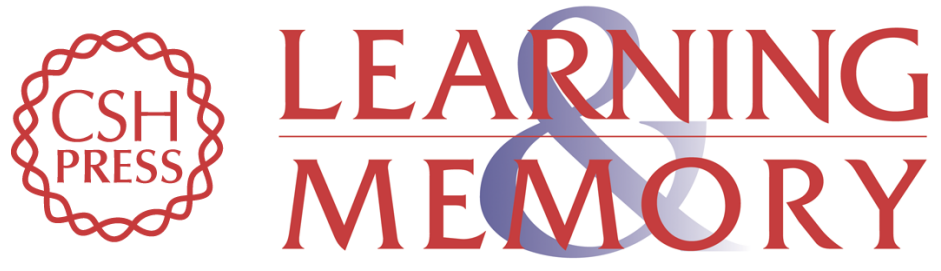

\section{A mutant mouse with a highly specific contextual fear-conditioning deficit found in an N-ethyl-N-nitrosourea (ENU) mutagenesis screen}

Leon G. Reijmers, Jennifer K. Coats, Mathew T. Pletcher, et al.

Learn. Mem. 2006, 13:

Access the most recent version at doi:10.1101/lm.98606

References This article cites 27 articles, 11 of which can be accessed free at:

http://learnmem.cshlp.org/content/13/2/143.full.html\#ref-list-1

License

Email Alerting Receive free email alerts when new articles cite this article - sign up in the box at the Service top right corner of the article or click here. 